\title{
Delimitação estatística dos lenhos juvenil e adulto de Eucalyptus e Corymbia
}

\section{Statistical delimitation of juvenile and mature wood of Eucalyptus and Corymbia}

\author{
Milene Teixeira Souzaㅁ, José Tarcísio Lima ${ }^{2}$, Bruno Charles Dias Soares ${ }^{3}$, \\ Selma Lopes Goulart ${ }^{4}$ e Lidiane Costa Lima ${ }^{3}$
}

\begin{abstract}
Resumo
Esse trabalho teve como objetivo delimitar a idade de transição entre o lenho juvenil e o adulto de Eucalyptus pilularis, Eucalyptus saligna, Eucalyptus cloeziana e Corymbia maculata com 37 anos de idade, a partir da análise das variações no sentido medula-casca do comprimento das fibras e do ângulo microfibrilar. Duas árvores de cada espécie foram selecionadas, abatidas e tiveram discos cortados na base do tronco. Amostras foram retiradas do disco no sentido medula-casca para a determinação do ângulo das microfibrilas e do comprimento das fibras. O E. saligna apresentou o maior comprimento médio das fibras e a menor média do ângulo microfibrilar. As idades de transição estimadas pela análise da variação do comprimento das fibras foram menores do que aquelas estimadas pela análise da variação do ângulo microfibrilar. A análise de regressão linear response plateau permitiu estimar o ponto em que ocorre a estabilização do comprimento das fibras e do ângulo microfibrilar para as espécies avaliadas.
\end{abstract}

Palavras-chave: Comprimento da fibra, ângulo microfibrilar, variação radial.

\begin{abstract}
The aim of this work was to determine the limit between juvenile and mature wood in Eucalyptus pilularis, Eucalyptus saligna, Eucalyptus cloeziana and Corymbia maculata at the age of 37 years, based on the pith to bark analysis of the variation of fibers length and microfibril angle. Two trees of each species were cut and $10 \mathrm{~cm}$ thick discs were removed from the base of the trunk. Samples were drawn in the pith-bark direction in the discs to determine the variation of microfibril angle and fiber length. $E$. saligna presented the highest mean fiber length and the smallest mean microfibril angle. The transition ages, estimated by analyzing the radial variation of the fibers length, were smaller than those estimated by the analysis of the microfibril angle variation. The response plateau analysis of regression allowed estimating the point at which stabilization of fiber length and microfibril angle occurs for the species analyzed.
\end{abstract}

Keywords: Fiber length, microfibril angle, radial variation.

\section{INTRODUÇÃO}

A madeira é um produto natural e renovável, porém a heterogeneidade do seu lenho dificulta o seu processamento e emprego na indústria. Dessa forma, o conhecimento de suas propriedades é de fundamental importância para sua correta utilização.

As espécies pertencentes aos gêneros Eucalyptus, exóticas à flora brasileira, têm sido utilizadas pela indústria madeireira em substituição de madeiras nativas (PELOZZI et al., 2012). O gênero Eucalyptus é um dos mais plantados em todo o mundo, abrangendo grande número de espécies que são utilizadas em diversos setores da indústria.

Segundo Vidaurre et al. (2011), com o decréscimo do suprimento de árvores adultas com grandes diâmetros que no passado eram oriundas de florestas naturais, houve o interesse na produção de árvores em ciclos curtos de corte por meio da adoção de espécies de rápido crescimento. Devido às crescentes pressões por demanda de madeira e mudanças nas práticas silviculturais despertou-se o

\footnotetext{
${ }^{1}$ Doutora em Ciência e Tecnologia da Madeira. UFLA - Universidade Federal de Lavras. Campus UFLA - 372000-000 Lavras, MG, Brasil. E-mail: tsmilene@gmail.com

${ }^{2}$ Professor Titular do Departamento de Ciências Florestais. UFLA - Universidade Federal de Lavras. Campus UFLA 372000-000 - Lavras, MG, Brasil. E-mail: jtlima@dcf.ufla.br

${ }^{3}$ Doutorando(a) em Ciência e Tecnologia da Madeira. UFLA - Universidade Federal de Lavras. Campus UFLA - 372000000 - Lavras, MG, Brasil. E-mail: bruno.soares18@hotmail.com, lidy_nanni@hotmail.com

${ }^{4}$ Professora Doutora. UFRA- Universidade Federal Rural da Amazônia / Campus Parauapebas. Caixa-postal: 3017 68515970 - Parauapebas, PA, Brasil. E-mail: lopesgoulart@yahoo.com.br
}

Sci. For., Piracicaba, v. 45, n. 116, p. 611-618, dez. 2017 DOI: dx.doi.org/10.18671/scifor.v45n116.02 
interesse em se estudar características anatômicas da madeira juvenil e sua influência nas propriedades físico-mecânicas da madeira (LARA PALMA; BALLARIN, 2003).

Ramay e Briggs (1986) definiram o lenho juvenil como sendo o xilema secundário, formado durante a fase jovem do câmbio vascular, nos primeiros anos de vida da árvore. Esse período varia conforme a espécie e pode ser afetado pelas condições ambientais. Assim, o lenho juvenil corresponde a uma região central no caule de forma cilíndrica, estendendo-se desde a base até o topo, podendo formar parte do alburno ou do cerne se este já estiver formado no tronco (ZOBEL; VAN BUIJTENEN, 1989).

A madeira jovem, normalmente se caracteriza por menor densidade, maior ângulo das microfibrilas na camada $\mathrm{S}_{2}$ da parede celular, fibras mais curtas, paredes celulares mais finas, maior conteúdo de lignina e hemicelulose, menor conteúdo de celulose e menor resistência em relação à madeira adulta (BENDTSEN, 1978; ZOBEL, 1984; LARA PALMA et al., 2010).

De acordo com Donaldson (2008) a retrabilidade ocorre na matriz da parede celular, com a saída de água abaixo do ponto de saturação das fibras. O mesmo autor afirma que as microfibrilas são ortogonais ao encolhimento da matriz e sua orientação explica em parte a natureza anisotrópica da retrabilidade. Assim, as paredes celulares com ângulo microfibrilar (AMF) muito baixo tendem a ter maiores contrações transversais, enquanto as paredes celulares com AMF muito alto tendem a ter maior contração longitudinal.

Segundo Palermo et al. (2013), as variações entre as propriedades da madeira dos lenhos juvenil e adulto podem interferir, de forma negativa, no processo de industrialização e transformação da madeira em outros produtos.

As características morfológicas de uma fibra, como comprimento e largura, são parâmetros importantes na estimativa da qualidade da polpa. O comprimento da fibra é mais importante propriedade para polpação, pois pode influenciar na resistência ao rasgo do papel. Marques et al. (2010) afirmaram que quanto maior for o tamanho das fibras, maior será a resistência do papel do rasgo. Por outro lado, fibras mais longas tendem a dar uma estrutura de folha mais aberta e menos uniforme.

As alterações anatômicas e químicas do lenho juvenil influenciam diretamente no comportamento mecânico da madeira, mas não se pode afirmar até que ponto a presença de madeira juvenil comprometerá a resistência mecânica da peça em uso (VIDAURRE et al., 2011). Segundo a mesma autora as diferenças entre o lenho juvenil e adulto são menos evidenciadas em madeiras de folhosas, mas estão sempre presentes nas espécies estudadas. Além disso, a origem genética, as condições de crescimento, bem como os tratos silviculturais em que a árvore se desenvolve influenciam diretamente na formação dos lenhos juvenil e adulto, e, consequentemente, nas propriedades da madeira.

Portanto, definir a idade de transição do lenho juvenil para o adulto torna-se importante, visto que as espécies de rápido crescimento são amplamente difundidas no Brasil e essa informação permite estimar melhor a qualidade e o aproveitamento da madeira de crescimento rápido para melhores utilizações (BENDTSEN; SENFT, 1986).

Nesse contexto, o objetivo deste trabalho foi delimitar a idade de transição entre o lenho juvenil e o adulto de Eucalyptus pilularis, Eucalyptus saligna, Eucalyptus cloeziana e Corymbia maculata com 37 anos de idade, a partir da análise das variações no sentido medula-casca do comprimento das fibras e do ângulo microfibrilar.

\section{MATERIAL E MÉTODOS}

\section{Procedência e coleta do material}

Árvores pertencentes às espécies Eucalyptus pilularis, Eucalyptus saligna, Eucalyptus cloeziana e Corymbia maculata foram selecionadas e abatidas, duas de cada espécie, com 37 anos de idade. $\mathrm{O}$ material foi proveniente do plantio experimental da Universidade Federal de Lavras, localizada na cidade de Lavras, Minas Gerais, latitude $21^{\circ} 14^{\prime} 43^{\prime \prime}$ S, longitude 44 $59^{\prime}$ 59" W e altitude de 919 m.

Foram mensurados altura e diâmetro de cada indivíduo selecionado, o que pode ser visto na Tabela 1. 
Tabela 1. Circunferência a $1,30 \mathrm{~m}$ de altura do solo (CAP), altura total da árvore (HT) e diâmetro a $1,30 \mathrm{~m}$ de altura do solo (DAP) das árvores de Eucalyptus pilularis, E. saligna, E. cloeziana e Corymbia maculata com 37 anos.

Table 1. Circumference at $1.30 \mathrm{~m}$ above the ground (CAP), total height of the tree (HT) and diameter at $1.30 \mathrm{~m}$ above the ground (DAP) of Eucalyptus pilularis, E. saligna, E. cloeziana and Corymbia maculata trees at 37 years old.

\begin{tabular}{lcccc}
\hline Espécie & Árvore & CAP $(\mathbf{m})$ & HT $(\mathbf{m})$ & DAP $(\mathbf{m})$ \\
\hline \multirow{2}{*}{ E. pilularis } & 43 & 1,72 & 40,9 & 0,547 \\
& 61 & 1,33 & 37,4 & 0,423 \\
\hline \multirow{2}{*}{ C. maculata } & 113 & 1,82 & 43,7 & 0,579 \\
& 109 & 1,99 & 39,0 & 0,633 \\
\hline \multirow{2}{*}{ E. saligna } & 140 & 1,83 & 42,0 & 0,583 \\
& 186 & 1,92 & 43,0 & 0,611 \\
\hline \multirow{2}{*}{ E. cloeziana } & 423 & 1,86 & 27,7 & 0,592 \\
& 320 & 1,39 & 19,8 & 0,442 \\
\hline
\end{tabular}

De cada árvore foram retirados discos de aproximadamente $10 \mathrm{~cm}$ de espessura, cortados na base do tronco, que foram utilizados para obtenção de amostras retiradas no sentido medula-casca para a determinação da variação radial do ângulo microfibrilar e do comprimento das fibras.

Os discos foram lixados com auxílio de uma lixadeira elétrica contendo lixa $n^{\circ} 320$, antes da retirada das amostras, para possibilitar a contagem dos anéis de crescimento. Isso permitiu que se conhecesse em que posição radial, cada anel de crescimento estava localizado.

\section{Determinação do comprimento das fibras}

Para determinação do comprimento das fibras foi preparada uma solução de ácido acético glacial e peróxido de hidrogênio, na proporção de 1:1 (FRANKLIN, 1945), onde foram colocadas pequenas lascas de madeira retiradas em cada ponto amostrado. Os frascos contendo o macerado foram deixados em estufa a $60^{\circ} \mathrm{C}$ por 24 horas, sendo então o material dissociado lavado em água corrente até a completa retirada da solução residual. Após a lavagem, o material passou por um processo de coloração com safranina a 1\% em álcool 50\% (BERLYN; MIKSCHE, 1976). Lâminas provisórias contendo o material dissociado foram montadas em solução aquosa de glicerina, para medição do comprimento das fibras. Foram medidas 20 fibras por amostra com auxílio do microscópio Olympus BX 51, associado ao sistema de análise de imagens Image Pro Plus.

Após ter seus pressupostos testados e atendidos, a análise de variância (ANOVA) e o teste de médias de Tukey foram aplicados aos dados para verificar se existem diferenças entre os comprimentos das fibras das espécies analisadas.

\section{Determinação do ângulo microfibrilar}

Para a medição do ângulo microfibrilar (AMF) da parede celular das fibras, cortes tangencias de $7 \mu \mathrm{m}$ de espessura foram obtidos com o auxílio de um micrótomo de deslize. Os cortes foram colocados em frascos de vidro e cobertos com uma solução de ácido acético glacial e peróxido de hidrogênio, na proporção de 1:1 (FRANKLIN, 1945). Os frascos foram lacrados e deixados em estufa a $60^{\circ} \mathrm{C}$ durante 12 horas para que ocorresse a dissolução da lamela média e a individualização dos elementos anatômicos, formando um macerado que foi então lavado e armazenado em água destilada. Após isso foram montadas lâminas provisórias imersas em uma solução aquosa de glicerina (1:1). O AMF médio foi determinado através da técnica de microscopia de luz polarizada, empregada por Lima et al. (2004), realizando-se 20 medições por amostra.

Os dados de AMF foram analisados da mesma forma como feito para o comprimento das fibras.

\section{Determinação da idade de transição entre os lenhos juvenil e adulto}

Foi realizada a análise de regressão linear response plateau, que ajusta modelos descontínuos, ou seja, ajusta uma equação linear e um plateau para descrever, neste caso, a variação de características anatômicas. Essa análise possibilita a estimativa da idade em que ocorre a estabilização do comprimento das fibras e do ângulo microfibrilar no sentido medula-casca, sendo essa considerada a idade de transição entre os lenhos juvenil e adulto.

O modelo de regressão linear response plateau é definido pelas equações 1 e 2: 
Souza et al. - Delimitação estatística dos lenhos juvenil e adulto em quatro espécies de Eucalyptus e Corymbia

$$
\begin{aligned}
& Y=a X+b, \text { se } X<C \\
& Y=Z, \text { se } X \geq C
\end{aligned}
$$

Em que: "Y" = comprimento das fibras ou ângulo microfibrilar; " $\mathrm{X}$ " = idade cambial; " $\mathrm{a}$ " = coeficiente linear; " $\mathrm{b}$ " = intercepto; " $\mathrm{C}$ " é o ponto de interseção do modelo linear com o plateau; " $\mathrm{Z}$ " = comprimento das fibras ou ângulo microfibrilar quando se atinge o plateau.

\section{RESULTADOS E DISCUSSÃO}

\section{Comprimento das fibras e ângulo microfibrilar}

As tabelas 2 e 3 mostram dados de estatística descritiva e o teste de comparação de médias realizado para os materiais analisados.

Tabela 2. Estatística descritiva e comparação das médias dos comprimentos das fibras encontrados em $E$. saligna, E. pilularis, E. cloeziana e C. maculata com 37 anos.

Table 2. Descriptive statistics and comparison of the mean fiber lengths found in E. saligna, E. pilularis, E. cloeziana and C. maculata trees at 37 years.

\begin{tabular}{lccccc}
\hline \multirow{2}{*}{ Espécie } & \multicolumn{5}{c}{ Comprimento das fibras $(\boldsymbol{\mu}$ ) } \\
\cline { 2 - 5 } & Mínimo & Médio & Máximo & Desvio Padrão & CV\% \\
\hline E. saligna & 840 & $1105 \mathrm{~A}$ & 1232 & 98,27 & 8,89 \\
E. pilularis & 733 & $1061 \mathrm{~A}$ & 1320 & 157,52 & 14,84 \\
E. cloeziana & 754 & $1027 \mathrm{AB}$ & 1177 & 117,70 & 11,46 \\
C. maculata & 737 & $971 \mathrm{~B}$ & 1124 & 83,41 & 8,59 \\
\hline
\end{tabular}

Médias seguidas de mesma letra na coluna não diferem entre si pelo teste de Tukey a $5 \%$ de probabilidade de erro.

De acordo com a Tabela 2, a espécie Eucalyptus saligna apresentou o maior comprimento médio das fibras, enquanto o a espécie Corymbia maculata apresentou a menor média dessa característica anatômica. Esse resultado mostra que a primeira espécie é a mais recomendada para polpação, levando em conta a necessidade de obtenção de papel mais resistente ao rasgo.

Tomazello Filho (1987), afirma que, sob o aspecto tecnológico, a variação das dimensões das fibras relaciona-se com as propriedades físico-mecânicas da madeira e da celulose e papel. Dentre as propriedades influenciadas pela anatomia citam-se os diversos índices de resistência, o colapso das fibras, as ligações inter-fibras.

Calonego et al. (2005) analisaram diferentes alturas em árvores de Corymbia citriodora com 32 anos de idade e verificaram que o comprimento das fibras aumentou no sentido medula-casca e que a região de lenho juvenil ocorreu desde a medula até os primeiros $55 \mathrm{~mm}$.

Ramos et al. (2011), estudando o comprimento das fibras em Eucalyptus grandis com 23 anos de idade, encontraram diferenças significativas entre as regiões próximas à medula e aquelas próximas à casca, com médias de 950,43 $\mu \mathrm{m}$ na região da medula e $1148,15 \mu \mathrm{m}$ próximo a casca, mostrando tendência de aumento do comprimento das fibras na direção medula-casca.

Tabela 3. Estatística descritiva e comparação das médias dos ângulos microfibrilares encontrados em $E$. saligna, $E$. cloeziana, E. pilularis e C. maculata com 37 anos.

Table 3. Descriptive statistics and comparison of the means of the microfibril angles found in $E$. saligna, E. cloeziana, E. pilularis and C. maculata at 37 years.

\begin{tabular}{lccccc}
\hline \multirow{2}{*}{ Espécie } & Mínimo & Médio & Máximo & Desvio Padrão & CV\% \\
\cline { 2 - 5 } & 8,35 & $9,95 \mathrm{~A}$ & 13,35 & 1,52 & 15,30 \\
\hline E. saligna & 8,00 & $9,98 \mathrm{~A}$ & 13,00 & 1,32 & 13,26 \\
E. cloeziana & 8,25 & $11,96 \mathrm{~B}$ & 17,05 & 2,69 & 22,48 \\
E. pilularis & 8,80 & $12,11 \mathrm{~B}$ & 19,05 & 3,19 & 26,31 \\
C. maculata & 8,805 &
\end{tabular}

Médias seguidas de mesma letra na coluna não diferem entre si pelo teste de Tukey a $5 \%$ de probabilidade de erro.

A Tabela 3 mostra que a madeira de E. saligna apresentou a menor média do ângulo microfibrilar, sendo esse valor bem próximo ao encontrado para E. cloeziana. A madeira de C. maculata apresentou o maior ângulo microfibrilar médio, segundo o teste de Tukey. 
Menores ângulos microfibrilares são associados a maiores valores de resistência e rigidez da madeira (Donaldson, 2008). Lara Palma et al. (2010) em estudo com Corymbia citriodora encontrou maiores valores de comprimento de fibra na região de lenho adulto. Zobel (1984) relatou que a espessura da parece celular, densidade e a resistência da madeira também apresentaram os maiores valores na região de madeira adulta.

Ramos et al. (2011) analisaram o ângulo microfibrilar em três árvores de Eucalyptus grandis com 23 anos, encontrando ângulo microfibrilar médio de $30,24^{\circ}$ na região próxima à medula e de $23,79^{\circ}$ próximo à casca, sendo esse um comportamento semelhante ao encontrado neste trabalho. Os autores apontaram que a transição entre os lenhos juvenil e adulto ocorreu entre o quinto e o décimo primeiro ano após o plantio, tendo em vista a variação radial do AMF.

\section{Delimitação dos lenhos juvenil e adulto}

As Figuras 1 e 2 mostram o comportamento do comprimento das fibras e do ângulo microfibrilar em função da idade cambial. Além disso, mostra a equação da reta e o plateau ajustados para cada espécie estudada.

É possível observar na Figura 1 que o comprimento das fibras sofre aumento mais acentuado ao longo dos anéis de crescimento produzidos durante os anos iniciais de seu desenvolvimento, o que caracteriza a madeira formada pelo câmbio ainda em fase de maturação. Quando o câmbio atinge o estado de maturidade, tende a produzir fibras com dimensões semelhantes ao longo dos anéis de crescimento formados a partir de então, através da divisão e diferenciação de suas células iniciais, que agora atingiram as dimensões máximas e formas determinadas pelos fatores genéticos ambientais e interações respectivas a cada espécie.
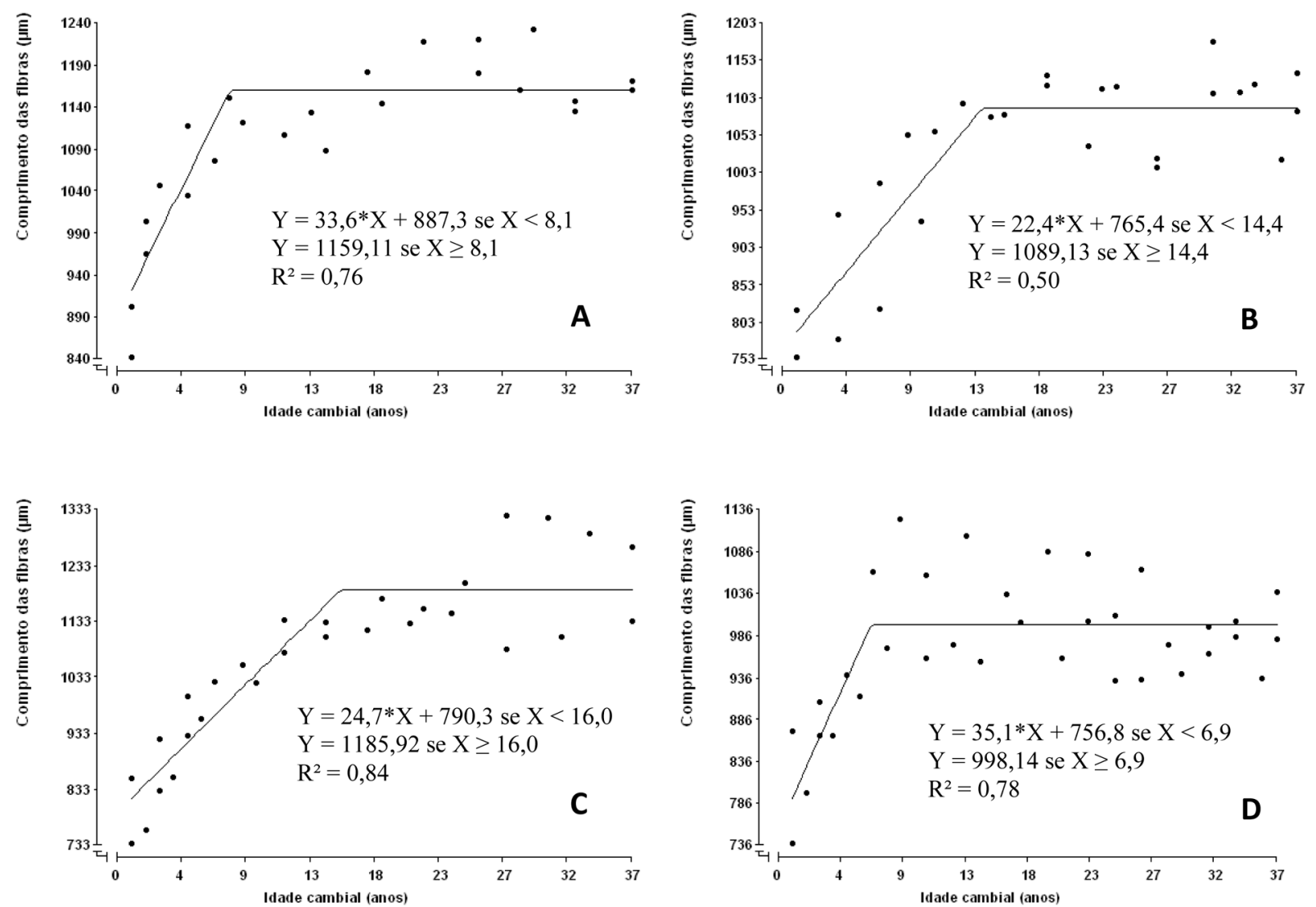

Figura 1. Comportamento do comprimento das fibras em função da idade em madeira de Eucalyptus saligna (A), E. cloeziana (B), E. pilularis (C) e C. maculata (D) com 37 anos.

Figure 1. Behavior of fiber length related to age in wood of Eucalyptus saligna (A), E. cloeziana (B), E. pilularis (C) and C. maculata (D) at 37 years.

As médias do comprimento das fibras foram menores na região próxima à medula para todas as espécies e tiveram uma tendência de aumento na direção da casca. Essa tendência de crescimento dos comprimentos de fibra também foi observada por Brasil e Ferreira (1972), Rocha et al. (2004), com Eucalyptus grandis, e Lara Palma et al. (2010) com Corymbia citriodora. 
Delucis et al. (2014) analisaram açoita-cavalo, nogueira-pecã e plátano e afirmaram ser o comprimento das fibras um bom parâmetro para determinação da idade de transição entre os lenhos juvenil e adulto. Os autores apontaram que a transição entre tais lenhos se deu entre 19 e 21 anos para açoita-cavalo, 14 e 16 anos para nogueira-pecã e 14 anos para plátano.
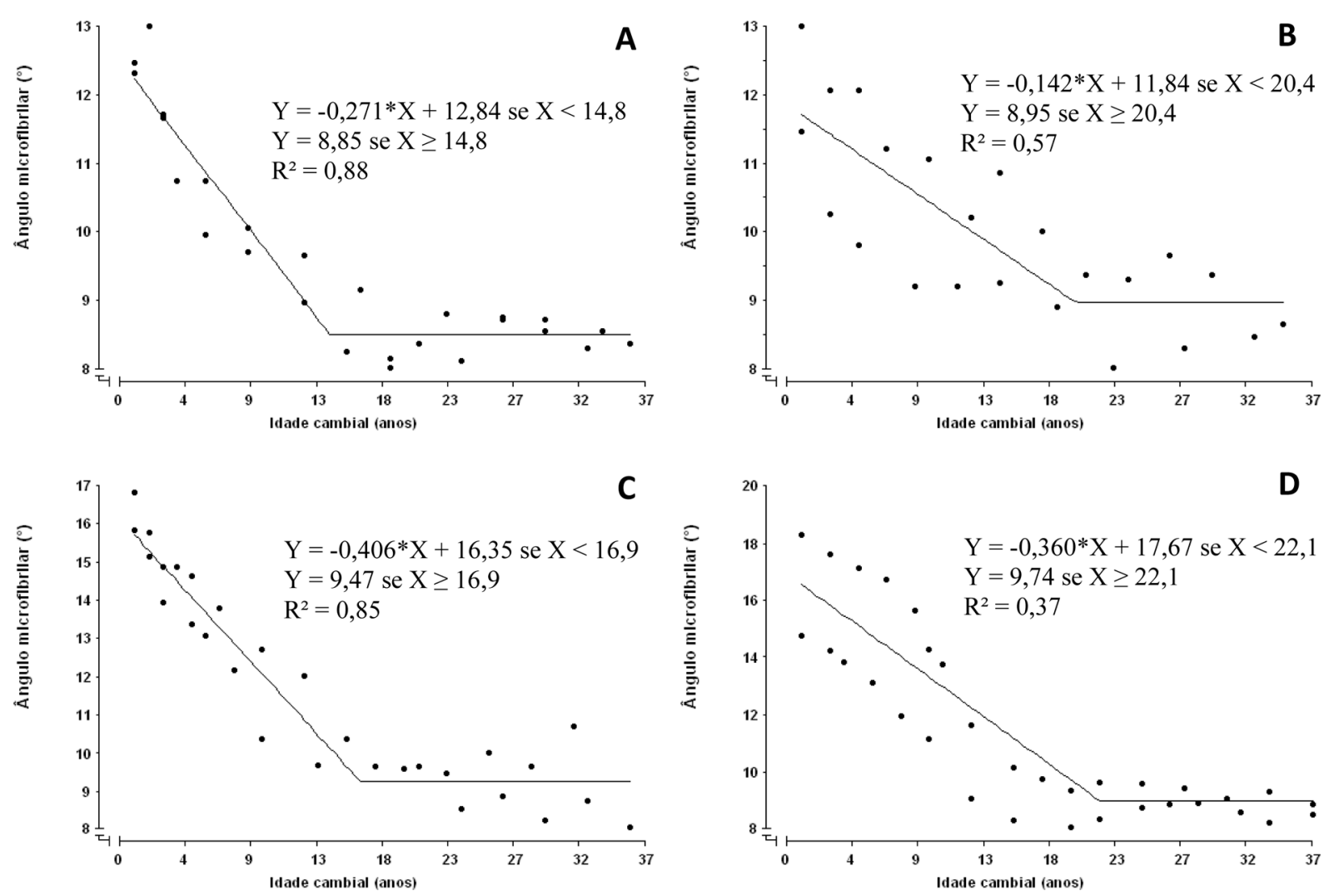

Figura 2. Comportamento do ângulo microfibrilar em função da idade cambial em madeira de Eucalyptus saligna (A), E. cloeziana (B), E. pilularis (C) e Corymbia maculata (D) com 37 anos.

Figure 2. Behavior of the microfibril angle related to the cambial age in wood of Eucalyptus saligna (A), E. cloeziana (B), E. pilularis (C) and Corymbia maculata (D) at 37 years.

De acordo com a Figura 2, maiores ângulos microfibrilares foram encontrados próximos à medula, que corresponde à porção juvenil do lenho. Esses valores diminuem à medida que a amostragem se aproxima da casca, região esta que é correspondente ao lenho adulto. Esse comportamento foi comum às espécies estudadas.

A regressão linear response plateau mostrou que a espécie E. saligna apresenta maior produção de lenho adulto, onde há pequena variação da medula para a casca do ângulo microfibrilar. De acordo com esse resultado, é possível inferir que essa é a espécie que menos sofreria com rachaduras e empenamentos decorrentes de contrações desiguais dos lenhos juvenil e adulto.

O coeficiente de determinação $\left(\mathrm{R}^{2}\right)$ observado no gráfico de C. maculata na Figura 2 é considerado baixo em relação às demais espécies. Isso pode ser explicado pela clara diferença vista no gráfico $\mathrm{D}$ entre os ângulos microfibrilares das duas árvores analisadas.

As idades de transição entre os lenhos juvenil e adulto estimadas com auxílio da análise de regressão linear response plateau para os dados de ângulo microfibrilar e comprimento de fibras das quatro espécies encontram-se na Tabela 4.

Observa-se na Tabela 4 que as idades de transição estimadas pela análise da variação radial do comprimento das fibras foram menores do que aquelas estimadas pela análise da variação do ângulo microfibrilar, com exceção das idades semelhantes encontradas para E. pilularis. Esses resultados indicam que a estabilização do ângulo das microfibrilas ocorre mais tarde do que a estabilização do comprimento das fibras.

A análise de correlação de Pearson entre ângulo microfibrilar e comprimento das fibras das amostras apresentou coeficiente de correlação igual a -0,616, significativo em nível de $1 \%$ de probabilidade de erro. De acordo com a classificação descrita por Dancey e Reidy (2006), a correlação 
entre as duas características é moderada. Isso mostra que o ângulo microfibrilar varia de forma inversa ao comprimento das fibras de modo que, no sentido medula-casca, o aumento do comprimento das fibras associa-se com a redução do ângulo microfibrilar em apenas $62 \%$ das vezes, o que justifica as diferentes idades de transição estimadas a partir da análise dessas características.

Tabela 4. Idades de transição entre os lenhos juvenil e adulto estimadas pela análise de regressão linear response plateau do ângulo microfibrilar e do comprimento das fibras de Eucalyptus pilularis, E. cloeziana, E. saligna e Corymbia maculata com 37 anos.

Table 4. Transition age between juvenile and adult wood estimated by the response plateau linear regression analysis of the microfibril angle and the fiber length of Eucalyptus pilularis, E. cloeziana, E. saligna and Corymbia maculata at 37 years.

\begin{tabular}{lcccc}
\hline Espécie & Comprimento das fibras $(\boldsymbol{\mu m})$ & Idade $($ anos) & Ângulo microfibrilar $\left(^{\circ}\right)$ & Idade $($ anos) \\
\hline E. pilularis & 1186 & 16 & 9,5 & 17 \\
C. maculata & 998 & 7 & 9,7 & 22 \\
E. cloeziana & 1089 & 14 & 8,9 & 20 \\
E. saligna & 1159 & 8 & 8,8 & 15 \\
\hline
\end{tabular}

\section{CONCLUSÕES}

O estudo da variação do comprimento das fibras e do ângulo microibrilar de E. saligna, E. cloeziana, E. pilularis e Corymbia maculata com 37 anos de idade permite concluir que:

O comprimento das fibras dessas espécies aumenta no sentido medula-casca e o ângulo microfibrilar sofre redução no mesmo sentido.

A análise de regressão linear response plateau mostrou que o ponto em que ocorre a estabilização do comprimento das fibras foi aos 7 e 8 anos para C. maculata e E. saligna, 14 e 16 anos para E. cloeziana e E. pilularis respectivamente. Para o ângulo microfibrilar o ponto de estabilização ocorre entre 15 e 22 anos de idade para as quatro espécies.

Os pontos em que o comprimento das fibras e o ângulo microfibrilar se estabilizam são diferentes. Isso faz com que a idade de transição entre os lenhos juvenil e adulto estimada pela análise do comprimento das fibras seja diferente da idade estimada pelo estudo do ângulo microfibrilar.

\section{REFERÊNCIAS BIBLIOGRÁFICAS}

BRASIL, M. A. M.; FERREIRA, M. Variação da densidade básica e das característcas das fibras em Eucalyptus grandis Hill ex Maiden ao nível do DAP. Revista IPEF, n. 5, p. 81-90, 1972.

BENDTSEN, B. A. Properties of wood from improved and intensively managed trees. Forest Products Journal, Madison, v. 28, n. 10, p. 69-72, 1978.

BENDTSEN, B. A.; SENFT, J. Mechanical and anatomical properties in individual growth rings of plantationgrown cottonwood and loblolly pine. Wood Fiber Science, Madison, v. 18, n.1, p. 23-28, 1986.

BERLYN, G. P.; MIKSCHE, J. P. Botanical microtechnique and cytochemistry. Iowa: State University Press, 1976. $326 \mathrm{p}$.

CALONEGO, F. W.; SEVERO, E. T. D.; ASSI, P. P. Mensuração do comprimento das fibras para a determinação da madeira juvenil de Eucalyptus citriodora. Scientia Forestalis, Piracicaba, n. 68, p. 113-121, 2005.

DANCEY, C.; REIDY, J. Estatística Sem Matemática para Psicologia: usando SPSS para Windows. Porto Alegre: Artmed, 2006.

DELUCIS, R. A.; GATTO, D. A.; STANGERLIN, D. M.; BELTRAME, R. Métodos de delimitação dos lenhos juvenil e adulto de três folhosas e propriedades biométricas de suas fibras. Revista Árvore, Viçosa, v. 38, n. 5, p. 943-950, 2014.

Sci. For., Piracicaba, v. 45, n. 116, p. 611-618, dez. 2017 DOI: dx.doi.org/10.18671/scifor.v45n116.02 
Souza et al. - Delimitação estatística dos lenhos juvenil e adulto em quatro espécies de Eucalyptus e Corymbia

DONALDSON, L. Microfibril angle: measurement, variation and Relationships - a review. IAWA Journal, Leiden, v. 29, n. 4, p. 345-386, 2008.

FRANKLIN, G. L. Preparation of thin sections of synthetic resins and wood-resin composites, and a new macerating method for wood. Nature, London, v. 155, p. 55, 1945.

LARA PALMA, H. A.; LEONELLO, E. C.; BALLARIN, A. W. Demarcação da madeira juvenil e adulta de Corymbia citriodora. Cerne, Lavras, v. 16, supp., p. 114-148, 2010.

LARA PALMA, H. A.; BALLARIN, A. W. Propriedades de contração na madeira juvenil e adulta de Pinus taeda L. Scientia Forestalis, Piracicaba, v. 64, p. 13-22, 2003.

LIMA, J. T.; BREESE, M. C.; CAHALAN, C. M. Variation in microfibril angle in Eucalyptus clones. Holzforschung, Berlin, v. 58, n. 2, p. 160-166, 2004.

MARQUES, G.; RENCORET, J.: GUTIÉRREZ, A.; DEL RÍO, J. C. Evaluation of the Chemical Composition of Different Non-Woody Plant Fibers Used for Pulp and Paper Manufacturing. The Open Agriculture Journal, Sharjah, v. 4, p. 93-10, 2010.

PALERMO, G. P. M.; LATORRACA, J. V. F.; SEVERO, E. T. D.; NASCIMENTO, A. M.; REZENDE, M. A. Delimitação entre os lenhos juvenil e adulto de Pinus elliottii Engelm. Revista Árvore, Viçosa, v. 37, n. 1, p. 191-200, 2013.

PELOZZI, M. M. A.; SEVERO, E. T. D.; CALONEGO, F. W.; RODRIGUES, P. L. M.; PROPRIEDADES FÍSICAS DOS LENHOS JUVENIL E ADULTO DE Pinus elliottii Engelm var. elliottii e de Eucalyptus grandis Hill ex Maiden. Ciência Florestal, Santa Maria, v. 22, n. 2, p. 305-313, 2012.

RAMOS, L. M. A.; LATORRACA, J. V. F.; PASTRO, M. S.; SOUZA, M. T.; GARCIA R. A.; MONTEIRO, A. C. Variação radial dos caracteres anatômicos da madeira de Eucalyptus grandis W. Hill Ex Maiden e idade de transição entre lenho juvenil e adulto. Scientia Forestalis, Piracicaba, v. 39, n. 92, p. 411-418, dez. 2011.

RAMAY, W.; BRIGGS, D. Juvenile Wood: has it come of age? In: A TECHNICAL WORKSHOP: JUVENILE WOOD - WHAT DOES MEAN TO FOREST MANAGEMENT AND FOREST PRODUCTS. 1986., Madsion. Proceedings... Madison: Forest Products Reserch Society, 1986. p. 5-11.

ROCHA, F. T.; FLORSHEIM, S. M. B.; COUTO, H. T. Z. Variação das dimensões dos elementos anatômicos da madeira de árvores de Eucalyptus grandis Hill ex Maiden aos sete anos. Revista Instituto Florestal, São Paulo, v. 16, n. 1, p. 43-55, 2004.

TOMAZELLO FILHO, M. Variação radial da densidade básica em estrutura anatômica da madeira do Eucalyptus globulus, E. pellita, e E. acmenioides. Revista IPEF, Piracicaba, n. 36, p. 35-42, 1987.

VIDAURRE, G.; LOMBARDI, L. R.; OLIVEIRA, J. T. S.; ARANTES, M. D. C. Lenho juvenil e adulto e as propriedades da madeira. Floresta e Ambiente, Seropédica, v. 18, n. 4, p. 469-480, 2011.

ZOBEL, B. J.; VAN BUIJTENEN, J. P. Wood variation: its causes and control. New York: Springer-Verlag; 1989. $363 \mathrm{p}$.

ZOBEL, B. J. The changing quality of the world wood supply. Wood Science and Technology, New York, v. 18, n. 1, p.1-17, 1984.

Recebido em 29/12/2017

Aceito para publicação em 14/06/2017 